\title{
Fisioterapia na reabilitação de pacientes submetidos à cirurgia ortognática: revisão narrativa
}

\author{
Physiotherapy in the rehabilitation of patients that underwent orthognathic surgery: a \\ narrative review
}

Fisioterapia en la rehabilitación de pacientes sometidos a cirugía ortognática: revisión narrativa

\begin{abstract}
Karoline de Melo Magalhães ${ }^{1 *}$, Bruna Caroline Tomé Barreto ${ }^{1}$, Eduardo Otero Amaral Vargas ${ }^{1}$, Luciana Rougemont Squeff ${ }^{1}$, Luiz Felipe Cardoso de Araujo ${ }^{1}$, Amanda Cunha Regal de Castro', Eduardo Franzotti Sant'Anna', Mônica Tirre de Souza Araujo'.
\end{abstract}

\section{RESUMO}

Objetivo: Descrever e revisar como a cirurgia ortognática pode afetar o sistema estomatognático, evidenciando a importância da fisioterapia pós-operatória durante o período de reabilitação. Revisão bibliográfica: A cirurgia ortognática pode diminuir a amplitude dos movimentos mandibulares, diminuir a força dos músculos da mastigação, piorar a função velofaríngea e causar alterações neurosensoriais de diferentes níveis. Pacientes orientados a realizar fisioterapia após a cirurgia apresentaram melhoras mais significativas e mais rápidas em relação aos pacientes que não receberam essa orientação. Diversos protocolos de exercícios foram propostos, com diferentes momentos de início, períodos de duração e tipos de exercícios realizados. Considerações finais: Os benefícios da cirurgia ortognática são inquestionáveis, porém ela também gera sequelas pós-operatórias, especialmente na musculatura orofacial e nervos sensitivos da região. Embora não haja um consenso na literatura sobre quando começar ou que tipos de exercícios realizar na fisioterapia pós-cirúrgica, a grande maioria afirma que pacientes que a realizam apresentam melhora das funções musculares de forma mais rápida e mais eficiente.

Palavras-chave: Cirurgia ortognática, Fisioterapia, Cuidados pós-operatórios.

\begin{abstract}
Objective: To describe and to review how orthognathic surgery can affect the stomatognathic system, emphasizing the importance of postoperative physiotherapy during rehabilitation period. Bibliographic review: Orthognathic surgery can decrease the range of mandibular movements, decrease the strength of the masticatory muscles, worsen velopharyngeal function and cause different levels of neurosensory changes. Patients instructed to undergo physical therapy after surgery presented more significant and faster improvement compared to patients who did not receive this instruction. Several exercise protocols have been proposed, with different start times, duration periods and types of exercises performed. Final considerations: The orthognathic surgery's benefits are unquestionable, but this procedure may also generate postoperative sequelae, especially concerning the orofacial muscles and sensitive nerves in the region. Although there is no consensus in the literature regarding when to start or what types of exercises to perform in post-surgical
\end{abstract}

\footnotetext{
${ }^{1}$ Universidade Federal do Rio de Janeiro (UFRJ), Rio de Janeiro - RJ.

*E-mail: karolinemmagalhaes@hotmail.com
}

Coordenação de Aperfeiçoamento de Pessoal de Nível Superior - Brasil (CAPES), Código de Financiamento 001. Processo 88882.424820/2019-01. 
physiotherapy, most of them affirm that patients who perform them show improvement in muscle functions more quickly and more efficiently.

Key words: Orthognathic surgery, Physical therapy specialty, Postoperative care.

\section{RESUMEN}

Objetivo: Describir y revisar como la cirugía ortognática puede afectar el sistema estomatognático, destacando la importancia de la fisioterapia postoperatoria durante el período de rehabilitación. Revisión bibliográfica: La cirugía ortognática puede disminuir la amplitud de movimientos mandibulares, disminuir la fuerza de los músculos masticatorios, perjudicar la función velofaríngea y causar diferentes niveles de cambios neurosensoriales. Pacientes orientados a someterse a fisioterapia después de la cirugía mostraron mejoras más significativas y más rápidas en comparación con los pacientes que no recibieron esta orientación. Se han propuesto varios protocolos de ejercicio, con diferentes momentos de inicio, períodos de duración y tipos de ejercicios realizados. Consideraciones finales: Los beneficios de la cirugía ortognática son incuestionables, pero ella también puede generar secuelas postoperatorias, especialmente en los músculos orofaciales y nervios sensibles de la región. Aunque no exista consenso en la literatura sobre cuándo comenzar o qué tipo de ejercicios realizar en fisioterapia postoperatoria, la gran mayoría dice que los pacientes que la realizan muestran mejoría en las funciones musculares de forma más rápida y eficiente.

Palabras clave: Cirugía ortognática, Fisioterapia, Cuidados posoperatorios.

\section{INTRODUÇÃO}

Deformidades dentofaciais são resultado de crescimento deficiente ou exagerado dos ossos da maxila e/ou mandíbula. Quando esta deformidade não pode ser solucionada apenas com camuflagem ortodôntica, torna-se necessário o tratamento combinado à cirurgia ortognática, a fim de atender às necessidades estéticas e funcionais do paciente, o que pode ocorrer em, aproximadamente, $18 \%$ dos tratamentos ortodônticos (HSU LF, et al., 2021; PIAO Y, et al., 2016).

Hoje, as principais técnicas cirúrgicas incorporadas à cirurgia ortognática incluem osteotomia Le Fort I, osteotomia sagital bilateral e genioplastia óssea, porém, ela também pode incluir muitos outros procedimentos cirúrgicos realizados não só na mandíbula e maxila, mas também nas órbitas e base anterior do crânio. $\mathrm{A}$ maioria desses procedimentos são realizados intraoralmente e são estabilizados com titânio ou placas e parafusos biodegradáveis, minimizando ou eliminando a necessidade de fixação intermaxilar (NARAN S, et al., 2018; BELL RB, 2018). Entretanto, o tratamento cirúrgico apresenta diversas variáveis que têm capacidade de interferir na mobilidade mandibular, na fonação e função velofaríngea e percepção neurosensorial (BOYD SB, et al., 1991; CHANCHAREONSOOK N, et al., 2006; KARAS ND, et al., 1990).

A fisioterapia pós-operatória, por sua vez, pode reduzir essas complicações e auxiliar o restabelecimento da função muscular, melhorando a vascularização, diminuindo a fatigabilidade e aumentando a força e a massa muscular, aumentando o metabolismo de proteínas, e ainda restaurando a anatomia normal do tecido fibroso interno (STORUM KA e BELL WH, 1986).

Esta revisão da literatura teve, portanto, o objetivo de descrever como a cirurgia ortognática pode afetar o sistema estomatognático, evidenciando a importância da fisioterapia pós-operatória nesta reabilitação.

\section{REVISÃO BIBLIOGRÁFICA}

\section{Os efeitos da cirurgia ortognática nos músculos e nas funções}

Estudos evidenciaram que pacientes submetidos à cirurgia ortognática apresentaram diminuição da amplitude do movimento mandibular e dores na articulação temporomandibular (ATM) após a cirurgia (STORUM KA e BELL WH, 1984; ARAGON SB, et al., 1985; ARAGON SB e VAN SICKELS JE, 1987; 
AGBAJE J, et al., 2018). Hipomobilidade da ATM e trismo podem ocorrer após a cirurgia e requerem tratamento precoce com intuito de evitar outros distúrbios (LO LJ, et al., 2008).

$\mathrm{O}$ ato cirúrgico pode produzir traumas na maxila, mandíbula, dentição, ATM, músculos mastigatórios e tecidos moles associados. A resposta esperada ao trauma é uma limitação do movimento das estruturas lesadas, que representa o ponto final de uma resposta neuromuscular complexa para minimizar danos adicionais ao tecido (BOYD SB, et al., 1991). Embora uma diferença significativa no movimento mandibular não tenha sido observada após a osteotomia Le Fort I ou a osteotomia vertical intraoral do ramo, em relação ao grupo controle, houve uma tendência geral de movimento mandibular reduzido. A abertura maxilomandibular, protrusão e excursões laterais foram estatisticamente menores em pacientes após osteotomia sagital do ramo mandibular, em relação ao grupo controle (STORUM KA e BELL WH, 1984).

Recidivas após cirurgias de recuo mandibular são causadas parcialmente por fatores musculares mastigatórios, que podem ser atribuídos a uma falha em atingir um equilíbrio homeostático entre a função muscular e a forma esquelética. Um estudo demonstrou que pacientes que apresentam maior força de mordida do músculo masseter em máxima intercuspidação habitual possuem uma maior tendência a recidivas após a cirurgia para correção da Classe III. Também foi observado que os músculos temporal e masseter apresentaram, em todos os testes eletromiográficos, diminuição de suas forças no primeiro mês pósoperatório e as recuperaram seis meses após a cirurgia (KO EWC, et al., 2013). Entretanto, outros autores encontraram um aumento significativo na atividade muscular a partir de seis a oito meses pós-operatórios (KOBAYASHI T, et al., 2001; CELAKIL D, et al., 2018).

Outro estudo observou que, comparados a uma amostra controle, pacientes prognatas, candidatos a cirurgia ortognática, apresentavam maior tendência a mastigação unilateral. Após a cirurgia, a mastigação se tornou mais balanceada, próxima a dos pacientes controle. Entretanto, a taxa de padrões de movimentos mandibulares anormais, mesmo diminuindo após a cirurgia, permaneceu maior que a dos pacientes controle (NAKATA Y, et al., 2007).

Chanchareonsook N, et al. (2006), em sua revisão, incluiu estudos que avaliaram a articulação da fala e a função velofaríngea durante a fonação em pacientes submetidos à cirurgia ortognática, e os resultados variaram de nenhum impacto na fala e na função velofaríngea a alguma melhora na articulação, até claro declínio na função velofaríngea para aqueles que já exibiam sinais ou tinham características limítrofes de disfunção velofaríngea. Concluiu-se que, embora o avanço da maxila possa ter impacto positivo na aparência facial, oclusão e articulação, os pacientes devem ser esclarecidos que, ao submeter-se à cirurgia, existe risco de deterioração da função velofaríngea para a fala.

Danos ao nervo alveolar inferior podem ocorrer durante a cirurgia ortognática, causando alterações neurosensoriais de diferentes níveis. A percentagem de sítios com déficits neurossensoriais no pós-cirúrgico imediato e a gravidade desse déficit inicial é maior nos indivíduos submetidos a osteotomia sagital do ramo mandibular, seguido pelos grupos submetidos a osteotomias Le Fort I, genioplastia isolada e osteotomia vertical do ramo (KARAS ND, et al., 1990). Em uma amostra submetida a osteotomias sagitais bilaterais, $67 \%$ apresentaram algum grau de alteração uma semana após o procedimento. Apesar da maioria das alterações terem desaparecido entre três meses e um ano, 15\% continuaram a sentir alterações mesmo após um ano e meio da cirurgia (YOSHIDA T, et al., 1989).

\section{Efeitos da fisioterapia na reabilitação pós-cirúrgica}

A fisioterapia pós-cirúrgica tem se mostrado um efetivo coadjuvante na recuperação precoce das amplitudes mandibulares e atividades musculares. Teng TTY, et al. (2015), em um estudo prospectivo, avaliou pacientes submetidos à cirurgia ortognática bimaxilar para correção de Classe III esquelética, antes da cirurgia, seis semanas (T2) e seis meses após (T3), e notou diferenças significativas entre o grupo que recebeu orientação para a realização de exercícios mandibulares e o grupo controle. Em T2, o grupo experimental atingiu amplitude de abertura máxima de $78,3 \%$ dos valores pré-cirúrgicos, enquanto o grupo controle atingiu $63,2 \%$ dos valores pré-cirúrgicos. Em T3, a amplitude de abertura máxima atingiu 108,6\% dos valores pré-cirúrgicos no grupo experimental, e no grupo controle atingiu $93 \%$ dos valores pré-cirúrgicos. 
Os mesmos autores investigaram a atividade eletromiográfica dos músculos destes pacientes durante os mesmos períodos do estudo anterior. A amplitude da atividade mioelétrica dos músculos masseter e temporal anterior atingiu 72,6-121,3\% dos valores pré-cirúrgicos no grupo experimental em T2; no grupo controle, 37,5$64,6 \%$. Em T3, a amplitude da atividade mioelétrica atingiu 135,1-233,4\% dos valores pré-cirúrgicos do grupo experimental, e no grupo controle, atingiu 89,6-122,5\% (KO EWC, et al., 2015).

Prado DGA, et al. (2018), em seu estudo clínico randomizado, ofereceu terapia miofuncional orofacial após cirurgia ortognática para um grupo e para outro não. O grupo tratado apresentou melhora significativa no tipo de mastigação, tornando-a mais balanceada seis meses após a cirurgia. A percentagem de indivíduos com tonicidade do lábio inferior e mobilidade da língua adequadas no grupo tratado aumentou significativamente três meses e seis meses após a cirurgia.

Os resultados da eletromiografia mostraram diminuição da duração do ato e do ciclo mastigatório após seis meses em relação aos tempos de três meses e pré-operatório, para o grupo tratado. Além disso, os valores foram próximos aos controles, que não foram submetidos à cirurgia. Também foi observado um aumento no número de ciclos mastigatórios após seis meses em comparação ao pré-operatório, indicando mastigação mais rápida (PRADO DGA, et al., 2018).

Pacientes que foram submetidos à cirurgia de osteotomia sagital do ramo e a regime de reabilitação com fisioterapia recuperaram o valor máximo de abertura bucal após 6-12 semanas de reabilitação. Já o grupo controle, que foi submetido a mesma cirurgia, mas não recebeu fisioterapia pós-operatória, teve uma diminuição significativa da abertura de $12 \mathrm{~mm}, 6$ meses após a cirurgia. Apesar da força de mordida não ter diminuído após a cirurgia no grupo controle, o grupo experimental apresentou aumento da força após fisioterapia. Ambos os grupos apresentaram diminuição na fadiga muscular, porém o grupo experimental obteve uma melhora mais expressiva (STORUM KA e BELL WH, 1986).

A melhora no sorriso foi detectada quatro meses após a cirurgia ortognática em pacientes orientados a realizar exercícios de treinamento dos músculos faciais (HONG SO, et al., 2017). Um mês após a cirurgia, $68 \%$ dos pacientes apresentavam sorriso baixo, 32\% sorriso médio, e nenhum paciente apresentava sorriso alto. Quatro meses após a cirurgia, houve diminuição significativa de sorrisos baixos para $50 \%$ e aumento de sorrisos médios para $45 \%$ e de sorrisos altos para $5 \%$.

A percentagem de pacientes que apresentavam curvatura labial ascendente aumentou de $52 \%$ para $73 \%$, de curvatura labial reta permaneceu $18 \%$ entre os dois tempos e a de curvatura labial descendente diminuiu de $30 \%$ para $9 \%$. Antes da fisioterapia, os últimos dentes superiores visíveis eram os primeiros pré-molares superiores $(48 \%)$ e os caninos (39\%). Depois da fisioterapia, apesar do primeiro pré-molar superior permanecer como o mais prevalente (59\%), o segundo pré-molar superior $(36 \%)$ foi o segundo dente posterior mais visível. Entretanto, apesar destes resultados serem promissores, não houve amostra controle para comparação dos resultados (HONG SO, et al., 2017).

Gallerano G, et al. (2012), em um estudo sem grupo controle, avaliou 30 pacientes submetidos à cirurgia ortognática com resultados do tratamento considerados bons ou excelentes. Todos foram instruídos a realizar exercícios de fortalecimento muscular. Após a retirada dos aparelhos ortodônticos 4-6 meses após a cirurgia, os pacientes foram reavaliados e 19 deles continuaram a apresentar problemas funcionais, sendo submetidos a uma segunda fase de tratamento miofuncional. Destes, 17 apresentaram deglutição atípica, 2 postura posterior da língua, 6 postura anterior da língua, 11 postura inferior da língua, 7 problemas de articulação da fala e 13 desequilíbrio da musculatura perioral.

Somente dois pacientes não apresentaram melhora nos aspectos avaliados após as sessões extras de terapia miofuncional. A deglutição atípica persistiu em quatro pacientes e a postura inferior da língua, problemas de articulação da fala e desequilíbrio da musculatura perioral persistiram em três pacientes (GALLERANO G, et al., 2012).

Alguns autores avaliaram a percepção de sensações alteradas por pacientes submetidos à cirurgia ortognática em um estudo clínico randomizado (PHILLIPS C, et al., 2007; PHILLIPS C, et al., 2010). Um grupo recebeu orientações para realizar apenas exercícios de abertura bucal e outro, além destes exercícios, 
recebeu orientações para realizar retreinamento sensorial. Os dois grupos não diferiram significativamente no pós-cirúrgico em termos de nível de problema percebido de dor intraoral ou facial. A diferença entre os dois grupos em cada visita não foi significativa para sensações incomuns, embora a tendência fosse para o grupo de retreinamento sensorial ter uma probabilidade maior de relatar menos problemas.

Após seis meses, a probabilidade de um sujeito relatar menor problema ou nível de interferência relacionado à dormência ou diminuição da sensibilidade labial foi significativamente maior no grupo de retreinamento sensorial, aproximadamente o dobro do grupo que realizou apenas exercícios de abertura. Até dois anos após a cirurgia, o grupo de exercícios de abertura teve maior probabilidade de relatar interferência nas atividades diárias relacionadas à dormência e perda de sensibilidade labial do que o grupo de retreinamento sensorial. A diferença entre os dois grupos foi relativamente constante (PHILLIPS C, et al., 2007; PHILLIPS C, et al., 2010).

Indivíduos mais velhos e aqueles com elevado sofrimento psicológico antes da cirurgia relataram maiores incômodos relacionados a sensações faciais incomuns, dormência e perda de sensibilidade labial. Em um estudo preliminar de Essick GK, et al. (2007), estes autores já haviam constatado que, após a cirurgia, as pessoas retreinadas exibiram menos prejuízo do que as pessoas não retreinadas apenas na percepção de dois pontos, sugerindo que as pessoas retreinadas experimentaram ou interpretaram os estímulos táteis de forma diferente do que as pessoas não retreinadas.

\section{Protocolos de exercícios utilizados}

Diversos protocolos de exercícios para reabilitação pós-cirúrgica foram propostos pelos autores ao decorrer dos anos. Aragon SB e Van Sickels JE (1987) propuseram, para a melhora da abertura mandibular, uma técnica autoadministrada pelo paciente. Nas duas primeiras semanas após a cirurgia não haveria exercícios, da segunda à quarta semanas foram orientados a aumentar sua abertura com exercícios leves e sem assistência de amplitude de movimento com meta de 25-30 mm na quarta semana. Das semanas 4-8, o paciente foi instruído a incorporar a assistência do dedo para abertura com objetivo de atingir $40 \mathrm{~mm}$ de abertura.

Lo LJ, et al. (2008), desenvolveram um dispositivo exercitador da ATM para tratar hipomobilidades e trismos. O dispositivo consistia em braços intraorais inseridos entre os dentes superiores e inferiores, e um parafuso para abrir os braços. Os pacientes eram instruídos a girar o parafuso do dispositivo para abrir a mandíbula até sentir desconforto. A abertura deveria ser sustentada por 1-2 minutos e liberada, com repetições totalizando 20 minutos, 5 vezes ao dia. Exercícios extras eram recomendados caso a recuperação fosse mais rápida que o esperado, e o uso do dispositivo era interrompido quando não mais necessário.

Osborne JJ (1989) dividiu sua terapia em pré e pós-operatória, em que uma sessão seria programada antes da cirurgia a fim de coletar dados clínicos do paciente, além de avaliar amplitude de movimento e fornecer instruções pós-operatórias como possíveis dificuldades que podem ocorrer e cuidados necessários. No período pós-operatório imediato deveria ser aplicado gelo/calor, estimulação elétrica e ultrassom. Dois métodos de alongamento foram instruídos: no alongamento com o dedo, o polegar é cruzado sobre o dedo formando um "X", o dedo indicador é colocado na parte inferior dos dentes e o polegar na parte superior, e uma leve pressão é aplicada na abertura até sentir um alongamento. $O$ exercício deveria ser feito bilateralmente e mantido por 6 segundos com 3 repetições por, no mínimo, 6 vezes ao dia.

Quando o paciente apresentasse dificuldade em aumentar abertura desta forma, espátulas de madeira deveriam ser colocadas entre os molares até alcançar resistência, então mais uma espátula deveria ser inserida entre aquelas já em posição para aumentar o alongamento, e deveriam ser mantidos nessa posição por 3-5 minutos de cada lado. A autora propõe consultas a cada semana, dez dias ou duas semanas a depender do progresso para avaliar os exercícios (OSBORNE JJ, 1989).

Kato K, et al. (2012) a fim de estimular a função mastigatória, indicaram exercício com goma de mascar iniciada 6 meses após a cirurgia e conduzida por 5 minutos, 2 vezes ao dia por 90 dias. Trawitzki LVV, et al. (2006), para a terapia pré-operatória, trabalharam apenas a musculatura tonal nos casos em que foram observadas alterações musculares importantes. Os pacientes que referiram dor ou desconforto na ATM após 
a cirurgia foram orientados a manter dieta leve e mastigação bilateral simultânea. Todos os pacientes foram orientados a realizar os movimentos mandibulares com ênfase na recuperação desses movimentos de forma gradativa. Exercícios foram utilizados para fortalecer e melhorar a postura de lábios e língua, e trabalhos corretivos foram realizados na deglutição e mastigação quando observadas alterações nesses aspectos.

No estudo de Yang HJ, et al. (2020), os pacientes realizaram exercícios para abertura da boca, a partir de 3 semanas após a cirurgia, até atingir abertura normal $(>40 \mathrm{~mm})$. O exercício para abertura bucal foi realizado com o polegar e o indicador para abertura bucal máxima, protrusão máxima e excursão lateral por 5-10 minutos. Em seguida, o paciente repousava com a utilização do posicionador final por pelo menos 30 minutos. Da terceira à sexta semana, os pacientes usaram um exercitador de mordida confeccionado a partir da mordida em oclusão cêntrica na almofada de silicone amolecida em água quente. O exercício de fechamento bucal foi realizado mastigando o aparelho 200 vezes pela manhã, ao meio-dia e à noite. Imediatamente após o exercício, foram realizados intervalos com a utilização do posicionador final por pelo menos 30 minutos.

No estudo de Prado DGA, et al. (2018) o paciente recebeu orientações sobre trauma cirúrgico, edema facial, diminuição da sensibilidade e movimentos faciais, dieta, higiene oral e cuidados pós-operatórios. Após a cirurgia ortognática, foi aplicado protocolo de 10 sessões, uma por semana, começando 30 dias após a cirurgia, abordando a sensibilidade, tônus, mobilidade, adequação da postura de lábios e língua, treinamento e adequação das funções miofuncionais orofaciais.

O protocolo preconizado por Teng TTY, et al. (2015) e Ko EWC, et al. (2015) indicou que os pacientes deveriam evitar movimentos mandibulares na primeira semana pós-operatória. Da segunda à quarta semana, os pacientes deveriam praticar movimentos da mandíbula, incluindo 6 repetições de abertura bucal máxima ativa, por 30 segundos a cada repetição; 10 repetições de movimento de excursão lateral e movimento protrusivo e retrusivo, por 5 segundos.

A partir da quinta semana, os pacientes começam a realizar 6 repetições de abertura bucal máxima passiva usando depressores de língua para pressionar a mandíbula o máximo possível para baixo; 10 repetições de excursão lateral ativa; 10 repetições de movimento mandibular protrusivo e retrusivo; e 3 repetições de um exercício isométrico de mordida dos músculos mastigatórios por aperto em oclusão cêntrica por 10 segundos. Os pacientes neste estudo foram solicitados a praticar essa reabilitação física uma vez antes de cada refeição e pelo menos três vezes ao dia. Após cada prática, os pacientes podiam relaxar e massagear manualmente os músculos mastigatórios (TENG TTY, et al., 2015; KO EWC, et al., 2015).

Storum KA e Bell WH (1986) indicaram um protocolo iniciando a reabilitação de seus pacientes no dia da liberação da imobilização maxilomandibular, cerca de seis semanas após a cirurgia, com abertura suave, protrusão e movimento lateral. Após o exercício, elásticos de treinamento verticais foram colocados bilateralmente distais aos caninos. Quando a abertura da mandíbula melhorou, o exercício assistido de amplitude de movimento com as duas mãos foi substituído por amplitude de movimento passiva, e o exercício dinâmico foi introduzido. Este último foi realizado com o auxílio de um aparelho de resistência ao movimento para aplicar força controlada aos músculos mastigatórios durante a abertura e fechamento da mandíbula.

A reabilitação foi encerrada e os pacientes colocados em um programa de manutenção quando a abertura da mandíbula e os movimentos laterais e protrusivos atingiram os valores pré-operatórios ou quando o exercício continuado por quatro semanas consecutivas falhou em aumentar a amplitude do movimento mandibular (STORUM KA e BELL WH, 1986).

No estudo de Boyd SB, et al. (1991), os pacientes foram instruídos a consumir uma dieta com alto teor de proteína nas primeiras duas semanas com progressão para uma dieta mecanicamente macia com mastigação mínima nas duas semanas seguintes, avançando para dieta regular sem restrição após um mês. O movimento mandibular foi mantido dentro de limites sem dor ou com desconforto mínimo durante as primeiras duas semanas. Isso foi seguido por exercícios ativos de amplitude de movimento da musculatura mastigatória.

No final do primeiro mês pós-operatório, o exercício passivo na forma de alongamento por manipulação digital era usado se houvesse apenas melhora mínima na mobilidade mandibular. O exercício ativo neste estudo foi definido como o movimento mandibular ocorrendo exclusivamente como resultado da atividade 
muscular da mastigação. Em contraste, o exercício passivo foi definido como movimento mandibular assistido resultante de manipulação digital ou aparelhos de alongamento da mandíbula (BOYD SB, et al., 1991).

Exercícios de retreinamento sensorial e de abertura foram dados durante as sessões de treinamento em uma semana, um mês e três meses após a cirurgia, por três estudos. No programa de exercícios de abertura, na sessão de uma semana os pacientes foram orientados a realizar movimentos simples de abertura e fechamento, e para lateral direita e esquerda usando apenas os músculos da mandíbula segurando por alguns segundos e depois relaxar, apenas até ocorrer desconforto, mas não dor; na sessão de um mês deveriam realizar os mesmos exercícios alongando com os dedos. Na sessão de três meses, caso a abertura fosse maior ou igual a $35 \mathrm{~mm}$, os exercícios deveriam ser repetidos ocasionalmente, ou caso fosse menor, a frequência dos exercícios deveria ser aumentada (PHILLIPS C, et al., 2007; ESSICK GK, et al., 2007; PHILLIPS C, et al., 2010).

E para o retreinamento sensorial em uma semana o paciente deveria alternar entre toque simples e golpes com um pincel de maquiagem (treinamento de movimento) olhando no espelho e com os olhos fechados, na sessão de um mês deveria alternar entre golpes para cima e para baixo e para lateral direita e esquerda (treinamento de orientação), olhando no espelho e com os olhos fechados. Na sessão de três meses, deveria alternar entre golpes de baixo para cima e de cima para baixo (treinamento de direcionalidade), olhando no espelho e com os olhos fechados. Cada sujeito assistiu a uma fita de vídeo explicando e demonstrando os exercícios apropriados para aquela sessão (PHILLIPS C, et al., 2007; ESSICK GK, et al., 2007; PHILLIPS C, et al., 2010).

\section{CONSIDERAÇÕES FINAIS}

Apesar de serem inquestionáveis os benefícios promovidos pela cirurgia ortognática aos pacientes que a ela se submetem, como qualquer outro evento cirúrgico, ela também gera sequelas pós-operatórias especialmente na musculatura orofacial e nervos sensitivos da região. Embora não haja um consenso na literatura sobre quando começar ou que tipos de exercícios realizar na fisioterapia pós-cirúrgica, a grande maioria afirma que pacientes que a realizam apresentam melhora das funções musculares de forma mais rápida e mais eficiente.

\section{AGRADECIMENTOS E FINANCIAMENTO}

O presente trabalho foi realizado com apoio da Coordenação de Aperfeiçoamento de Pessoal de Nível Superior - Brasil (CAPES), Código de Financiamento 001 (Processo 88882.424820/2019-01).

\section{REFERÊNCIAS}

1. AGBAJE J, et al. Pain Complaints in Patients Undergoing Orthognathic Surgery. Pain Research and Management, $2018 ; 2018(15): 4235025$.

2. ARAGON SB, et al. The effects of orthognathic surgery on mandibular range of motion. Journal of Oral and Maxillofacial Surgery, 1985; 43:938-943.

3. ARAGON SB e VAN SICKELS JE. Mandibular range of motion with rigid/nonrigid fixation. Oral Surgery, Oral Medicine, Oral Pathology, 1987; 63:408-411.

4. BELL WH, et al. Muscular rehabilitation after orthognathic surgery. Oral Surgery, Oral Medicine, Oral Pathology, 1983; 56(3):229-235.

5. BELL RB. A History of Orthognathic Surgery in North America. Journal of Oral and Maxillofacial Surgery, 2018; 76:2466-2481.

6. BOYD SB, et al. Recovery of Mandibular Mobility Following Orthognathic Surgery. Journal of Oral and Maxill ofacial Surgery, 1991; 49:924-931.

7. CELAKIL D, et al. Effect of orthognathic surgery on masticatory performance and muscle activity in skeletal Class III patients. The Journal of Craniomandibular \& Sleep Practice, 2018; 36(3):174-180.

8. CHANCHAREONSOOK N, et al. The effect of cranio-maxillofacial osteotomies and distraction osteogenesis on speech and velopharyngeal status: a critical review. The Cleft Palate-Craniofacial Journal, 2006; 43:477-487. 
9. ESSICK GK, et al. Effect of Facial Sensory Re-training on Sensory Thresholds. Journal of Dental Research, 2007; 86(6):571-575.

10. GALLERANO G, et al. Myofunctional and speech rehabilitation after orthodontic-surgical treatment of dentomaxillofacial dysgnathia. Progress in Orthodontics, 2012; 13:57-68.

11. HONG SO, et al. Physical Therapy for Smile Improvement After Orthognathic Surgery. The Journal of Craniofacial Surgery, 2017; 28: 422-426.

12. HSU LF, et al. Differences of condylar changes after orthognathic surgery among Class II and Class III patients. Journal of the Formosan Medical Association, 2021; In press.

13. KARAS ND, et al. Recovery of neurosensory function following orthognathic surgery. Journal of Oral and Maxillofacial Surgery,1990; 48(2):124-134.

14. KATO K, et al. Changes in masticatory functions after surgical orthognathic treatment in patients with jaw deformities: Efficacy of masticatory exercise using chewing gum. Journal of Oral and Maxillofacial Surgery, Medicine, and Pathology, 2012; 24:147-151.

15. KO EWC, et al. Alteration of Masticatory Electromyographic Activity and Stability of Orthognathic Surgery in Patients with Skeletal Class III Malocclusion. Journal of Oral and Maxillofacial Surgery, 2013; 71:1249-1260.

16. KO EWC, et al. The effect of early physiotherapy on the recovery of mandibular function after orthognathic surgery for class III correction. Part II: Electromyographic activity of masticatory muscles. Journal of Cranio-Maxillo-Facial Surgery, 2015; 43:138-143.

17. KOBAYASHI T, et al. Changes in masticatory function after orthognathic treatment in patients with mandibular prognathism. British Journal of Oral and Maxillofacial Surgery, 2001; 39:260-265.

18. LO LJ, et al. A device for temporomandibular joint exercise and trismus correction: design and clinical application. Journal of Plastic, Reconstructive \& Aesthetic Surgery, 2008; 61(3):297-301.

19. NAKATA Y, et al. Changes in Stomatognathic Function Induced by Orthognathic Surgery in Patients with Mandibular Prognathism. Journal of Oral and Maxillofacial Surgery, 2007; 65:444-451.

20. NARAN S, et al. Current Concepts in Orthognathic Surgery. Plastic and Reconstructive Surgery, 2018; 141: 925e.

21. OSBORNE JJ. A Physical Therapy Protocol for Orthognathic Surgery. Cranio, 1989; 7(2):132-136.

22. PHILLIPS C, et al. Sensory Retraining After Orthognathic Surgery: Effect on Patients' Perception of Altered Sensation. Journal of Oral and Maxillofacial Surgery, 2007; 65:1162-1173.

23. PHILLIPS C, et al. Sensory retraining: burden in daily life related to altered sensation after orthognathic surgery, a randomized clinical trial. Orthodontics \& Craniofacial Research, 2010; 13:169-178.

24. PIAO Y, et al. Five-year investigation of a large orthodontic patient population at a dental hospital in South Korea. The Korean Journal of Orthodontics, 2016; 46(3):137-145.

25. PRADO DGA, et al. Effects of orofacial myofunctional therapy on masticatory function in individuals submitted to orthognathic surgery: a randomized trial. Journal of Applied Oral Science, 2018; 26: e20170164.

26. STORUM KA, BELL WH. Hypomobility after maxillary and mandibular osteotomies. Oral Surgery, Oral Medicine, Oral Pathology, 1984; 57(1):7-12.

27. STORUM KA, BELL WH. The Effect of Physical Rehabilitation on Mandibular Function after Ramus Osteotomies. Journal of Oral and Maxillofacial Surgery, 1986; 44:94-99.

28. TENG TTY, et al. The Effect of early physiotherapy on the recovery of mandibular function after orthognathic surgery for Class III correction: Part I - Jaw-motion analysis. Journal of Cranio-Maxillo-Facial Surgery, 2015; 43:131-137.

29. TRAWITZKI LVV, et al. Effect of treatment of dentofacial deformities on the electromyographic activity of masticatory muscles. International Journal of Oral and Maxillofacial Surgery, 2006; 35:170-173.

30. YANG HJ, et al. Effects of Chewing Exerciser on the Recovery of Masticatory Function Recovery after Orthognathic Surgery: A Single-Center Randomized Clinical Trial, a Preliminary Study. Medicina, 2020; 56(9):483.

31. YOSHIDA T, et al. Impairment of the inferior alveolar nerve after sagittal split osteotomy. Journal of CranioMaxillofacial Surgery, 1989; 17(6):271-277. 